\title{
Software and Hardware Solutions for Channel Estimation Based on Cyclic Golay Sequences
}

\author{
Barna CSUKA, Zsolt KOLLAR \\ Department of Broadband Infocommunications and Electromagnetic Theory, \\ Budapest University of Technology and Economics, Egry József Street 18, H-1111 Budapest, Hungary \\ \{csuka, kollar\}@hvt.bme.hu \\ Manuscript received March 16, 2016
}

\begin{abstract}
This paper presents channel estimation methods based on cyclic complementary Golay sequences. First, the conventional Golay correlator is investigated, then a frequency domain approach using Discrete Fourier Transform (DFT) is provided. A complex valued fast Golay correlator is introduced which can be used for the estimation of complex valued channel impulse response. Furthermore, this paper presents the Recursive DFT (R-DFT), a signal processing architecture which may be beneficial compared to the wellknown Fast Fourier Transform (FFT). The R-DFT is able to efficiently calculate a point-by-point block spectra of the input signal, which makes it suitable for hardware implementation. Throughout the paper, the R-DFT is applied and it is compared to the conventional estimation methods. Finally, the efficiency of the proposed schemes is compared through simulations based on the $60 \mathrm{GHz}$ WiGig and the COST 207 standard, applying various channel models.
\end{abstract}

\section{Keywords}

Channel estimation, Golay sequences, R-DFT

\section{Introduction}

Wireless transmissions using high data rates call for fast and accurate parameter estimation for compensation of the imparities in the transciever chain. Devices require algorithms with low complexity and high precision for reduced power consumption and elevated data rates.

The focus of the paper is the application of the complementary Golay sequences [1], [2] in digital transmission using complex baseband signals. Based on their advantageous correlation properties, powerful algorithms can be developed for the receivers to estimate and compensate imperfections [3], [4] or channel impulse response (CIR) [5]. Furthermore, the sequences are evenly distributed along the available spectrum. The conventional solution calculates correlations [5], and the sum of these correlations provides the CIR.

In this paper, the complex CIR estimation techniques are presented based on complex complementary Golay se- quences and the corresponding complex valued Golay correlator is discussed in detail. This solution has not yet been adequately described in the literature.

An alternative solution for CIR estimation is presented using the Discrete Fourier Transform (DFT) providing results similar to that of the Golay correlator. Furthermore, the paper focuses on the application of the Recursive DFT (RDFT) for signal processing in digital communication, which is a less known structure for calculating the DFT of a signal sequence in a real-time manner [6], [7]. In contrast with the conventional DFT filter structure [8], which can be unstable due to the quantization errors, this method is robust against stability issues. Until now the R-DFT structure was primary used for measurement purposes. For wireless communication, especially for Orthogonal Frequency Division Multiplexing (OFDM), the most frequently used solution for spectral analysis and parameter estimation is the Fast Fourier Transform (FFT). Despite the FFT being the most commonly used algorithm, solutions employing the R-DFT have been proposed for wireless communication [7], [9].

Following the estimation of the CIR the channel equalization can be performed. Two equalization methods are investigated: the zero forcing $(\mathrm{ZF})$ and the minimum mean square error (MMSE) channel equalization. The theoretical methods for CIR estimation are validated using various channel models. The first part of the simulations is performed using models of an indoor $60 \mathrm{GHz}$ channel [10] with a signal compliant the IEEE 802.11ad standard [11], which applies Golay sequences. The second part uses models of the COST 207 standard for radio applications [12].

The paper is organized as follows. Section 2 briefly describes the Golay sequences. Section 3 presents the CIR estimation techniques using correlation and the DFT with a comparison of their hardware requirements in Sec. 4. In Sec. 5 the simulation results are presented and evaluated. Finally, Sec. 6 draws the conclusions.

\section{Complementary Golay Sequences}

The Golay sequence pairs are such $N$ element long binary sequences with elements $\{-1,1\}$ for which $a[k]$ and 
$b[k]$ are complementary to one another [1]. This means that the sum of their autocorrelation functions $\left(R_{a}[k], R_{b}[k]\right)$ is zero, except when the discrete time index $k$ is zero:

$$
R_{a}[k]+R_{b}[k]= \begin{cases}1, & \text { if } k=0 \\ 0, & \text { if } k \neq 0\end{cases}
$$

where

$$
\begin{aligned}
& R_{a}[k]=\sum_{l=0}^{N-k-1} a[l] \cdot a[l+k], \\
& R_{b}[k]=\sum_{l=0}^{N-k-1} b[l] \cdot b[l+k] .
\end{aligned}
$$

The following algorithm is used for generating the corresponding Golay sequences with a length of $N$, where $N$ is a power of 2 [2]:

$$
\begin{aligned}
& a_{0}[k]=b_{0}[k]=\delta[k], \\
& a_{n}[k]=a_{n-1}[k]+\alpha_{n} \cdot b_{n-1}\left[k-D_{n}\right], \\
& b_{n}[k]=b_{n-1}[k]-\alpha_{n} \cdot b_{n-1}\left[k-D_{n}\right],
\end{aligned}
$$

where $\delta[k]$ denotes the Kronecker delta function, $n$ is the number of stages in the generator $\left(n_{\max }=\log _{2} N\right) . \alpha_{n}$ contains the multiplication coefficients, and $D_{n}$ is the number of the delay elements (see Fig. 1).

In this paper, the lengths of the two complementary Golay sequences are $N=[16,32,64,128]$, which are denoted by $g_{A}, g_{B}$. Their inverted versions are $-g_{A},-g_{B}$. If the length of the sequences is also marked, then they are referred to as $g_{A, 16}, g_{A, 32}, g_{A, 64}$ etc. The applied $\alpha_{n}$ and $D_{n}$ values for the generators are given in Tab. 1 .

\subsection{Channel Estimation Field (CEF)}

The transmitted signal is affected by the radio channel, so it is necessary to compensate the received data. However, the receiver has to know the CIR to perform this correction. Therefore the transmitter appends predefined sequences to the message as a preamble. The receiver knows this ideal, predefined (or even standardized) preamble and also its location in the message. This known preamble may be compared to the received one, and the transfer characteristic can then be estimated for compensation purposes.

In this paper, the structure of the applied preamble is built up from Golay sequences as shown in Fig. 2. This arrangement originates from the IEEE 802.11 ad standard [11], but it can be generalized for shorter sequences. The basic elements are $g_{A}$ and $g_{B}$, which are the building blocks of the CEF $g_{U}$ and $g_{V}$.

As shown in Fig. 2, this structure contains $-g_{B}$ as a cyclic element. This cyclic property allows the estimation of the CIR in a stationary state as long as the CIR is shorter than the cyclic element.

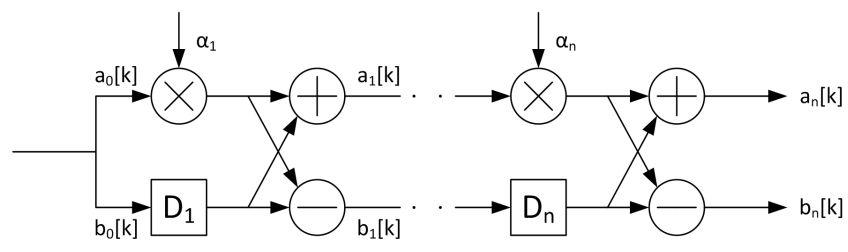

Fig. 1. Schematic structure of the Golay generator.

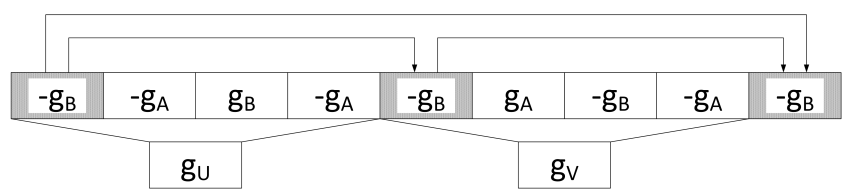

Fig. 2. Structure of the applied preamble.

\begin{tabular}{|c|c|c|}
\hline$N$ & $\alpha_{n}$ & $D_{n}$ \\
\hline 16 & {$[+1,+1,+1,-1]$} & {$[8,1,2,4]$} \\
\hline 32 & {$[-1,+1,-1,+1,-1]$} & {$[1,4,8,2,16]$} \\
\hline 64 & {$[+1,+1,-1,-1,+1,-1]$} & {$[2,1,48,16,32]$} \\
\hline 128 & {$[-1,-1,-1,-1,+1,-1,-1]$} & {$[1,8,2,4,16,32,64]$} \\
\hline
\end{tabular}

Tab. 1. Applied $\alpha_{n}$ and $D_{n}$ values.

\subsection{Complex Golay Sequences}

Nowadays, complex-valued IQ digital transmission is commonly used. However, if complex-valued transmission is applied, the channel model has to be also considered as complex-valued. To achieve complex channel estimation, the estimator sequences have to be complex-valued as well.

The CEF is given for real-valued transmission channels in Sec. 2.1, but it can be expanded to a complex-valued case as well. The idea originates from the IEEE 802.11ad standard [11], where a $\pi / 2$-rotation is applied. It can be utilized by multiplying the signal stream sample by sample with the following time function: $\mathrm{e}^{\mathrm{j} k \frac{\pi}{2}}(\mathrm{j}=\sqrt{-1})$. This rotation has to be handled at the Golay generator and complex sequences have to be generated so the $\alpha_{n}$ values are modified as follows:

$$
\alpha_{n}^{\prime}=\alpha_{n} \cdot \mathrm{e}^{-\mathrm{j} \frac{\pi}{2} D_{n}}
$$

If $D_{n}=1$, then the corresponding $\alpha_{n}$ value has to be multiplied by $-\mathrm{j}$. If $D_{n}=2$, then $\alpha_{n}$ has to be multiplied by -1 . The rest of the $\alpha_{n}$ values remain unaltered as the multiplication factor is 1 . These modifications circularly rotate the generated sequences on the complex plane by $\pi / 2$. The modified $\alpha_{n}$ values are shown in Tab. 2. (The $D_{n}$ values remain unaltered.)

\begin{tabular}{|c|c|}
\hline$N$ & $\alpha_{n}^{\prime}$ \\
\hline 16 & {$[+1,-\mathrm{j},-1,-1]$} \\
\hline 32 & {$[+\mathrm{j},+1,-1,-1,-1]$} \\
\hline 64 & {$[-1,-\mathrm{j},-1,-1,+1,-1]$} \\
\hline 128 & {$[+\mathrm{j},-1,+1,-1,+1,-1,-1]$} \\
\hline
\end{tabular}

Tab. 2. Applied $\alpha_{n}^{\prime}$ values for complex-valued sequences. 


\section{Estimation and Compensation of the Channel Impulse Response}

\subsection{Baseband Channel Model}

A baseband-equivalent channel model is applied for the simulations. The transmitted signal $(x[k])$ is filtered by the CIR. The channel is modeled as a FIR-filter with impulse response $h[k]$. Furthermore, the noise of the channel $(w[k])$ is assumed to be an additive, white Gaussian noise. As a result, the received signal $(y[k])$ is described as follows:

$$
y[k]=x[k] * h[k]+w[k] .
$$

\subsection{Estimation in the Time Domain}

In this paper, the complex Golay sequences $g_{A}$ and $g_{B}$ are applied. If the incoming CEF contains $g_{U}$ and $g_{V}$ sequences, then the sum of their correlations by $g_{A}$ and $g_{B}$ gives the CIR, as long as no noise is present in the channel. The structure of the procedure is presented in Fig. 3.

If the current $x[k]$ is $g_{A}[k]$, then the received signal is $g_{A}[k] * h[k]$, and the correlated signal can be expressed as

$$
R_{a}[k]=g_{A}[k] * g_{A}[k] * h[k] .
$$

Similarly, if $x[k]$ is $g_{B}[k]$, then the received and correlated signal is

$$
R_{b}[k]=g_{B}[k] * g_{B}[k] * h[k] .
$$

Sum of the shifted versions of (7) and (8) results in the estimated CIR, $\widetilde{h}[k]$, which can be expressed as

$$
\begin{aligned}
& \widetilde{h}[k]=R_{a}[k]+R_{b}[k], \\
& \widetilde{h}[k]=\left(g_{A}[k] * g_{A}[k]+g_{B}[k] * g_{B}[k]\right) * h[k] .
\end{aligned}
$$

As the $g_{A}$ and $g_{B}$ sequences are complementary to each other, therefore (10) can be simplified according to (1), which results in $\widetilde{h}[k]=h[k]$.

\subsection{Estimation in the Frequency Domain}

Another technique for channel estimation can be obtained with the aid of the DFT in the frequency domain. The estimated spectral values of the incoming Golay sequences corresponding to $y_{u}$ and $y_{v}$ are $Y_{U}$ and $Y_{V}$. The theoretical spectral values $\left(G_{U}\right.$ and $\left.G_{V}\right)$ for the corresponding transmitted Golay sequences $g_{v}$ and $g_{u}$ are calculated and stored previously as reference values. The channel transfer function can be calculated using the DFT of the CIR:

$$
H[i]=\sum_{k=0}^{N-1} h[k] \cdot \mathrm{e}^{\mathrm{j} \frac{2 \pi}{N} i k}
$$

where $i$ denotes the spectral bins $(i=0,1, \ldots N-1)$.

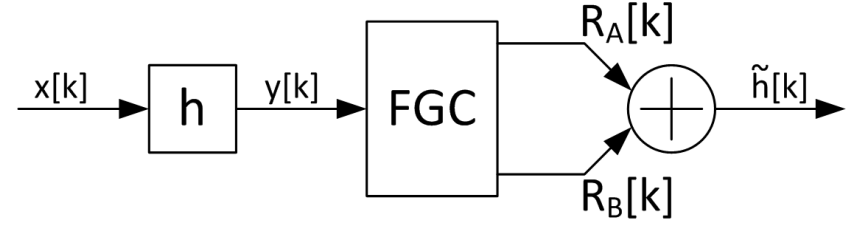

Fig. 3. Principle of channel estimation using correlation.

The transfer function can then be estimated in the frequency domain as

$$
\begin{aligned}
\widetilde{H}_{U}[i] & =Y_{U}[i] / G_{U}[i], \\
\widetilde{H}_{V}[i] & =Y_{V}[i] / G_{V}[i] .
\end{aligned}
$$

These estimates can be compared, and the estimated transfer function $(\widetilde{H}[i])$ is given by averaging $\widetilde{H}_{U}[i]$ and $\widetilde{H}_{V}[i]$. Finally, the inverse Fourier transform of $\widetilde{H}[i]$ provides the estimate of the CIR. It is important to note that some spectral values in $G_{U}$ and $G_{V}$ can be zero. To avoid the division by zero, the corresponding value has to be interpolated or replaced by the value of an another channel estimate.

This calculation procedure is the same with R-DFT (Sec. 4.2). If the last measured data of $g_{U}$ or $g_{V}$ is received, then the R-DFT has the correct value of $Y_{U}$ or $Y_{V}$ at the output. To satisfy the convergence criteria of R-DFT [6], $N$ has to be greater than or equal to the length of $g_{U}$ or $g_{V}$.

\subsection{Compensation of the Channel Fading Ef- fects}

If the channel transfer function (Sec. 3.2-3.3) and the Signal to Noise Ratio (SNR) are known [5], then a compensation filter can be created to filter the distortions of the radio channel. This compensation filter $\left(H_{\text {comp }}\right)$ has to fulfill the following criterion: $H[i] \cdot H_{\text {comp }}[i] \approx 1$. The simplest compensation is the $\mathrm{ZF}$ equalization:

$$
H_{\mathrm{ZF}}[i]=\frac{1}{\widetilde{H}[i]} .
$$

This is a simple calculation, but it can cause errors: if the transfer function at the given frequency is very small, then the amplifying of the compensation is very large, and not only the signal, but the noise is amplified as well. The MMSE equalization technique suppresses these errors, and uses the estimated SNR $(\widetilde{\eta})$ for the compensation as well [5]. The transfer function of the MMSE channel equalizer can be expressed as follows:

$$
H_{\text {MMSE }}[i]=\frac{\overline{\widetilde{H}[i]}}{|\widetilde{H}[i]|^{2}+1 / \widetilde{\eta}}
$$

where $\overline{\widetilde{H}[i]}$ denotes the complex conjugate of $\widetilde{H}[i]$. The $1 / \widetilde{\eta}$ term prevents the over-amplification of the noise. 


\section{Hardware Solutions for CIR Esti- mation}

\subsection{Fast Golay Correlator (FGC)}

The FGC [2] is the conventional solution to calculate the correlations with the sequences $g_{A}$ and $g_{B}$. This correlator has the same description and structure as the generator in Fig. 1, but the initial values of $a_{0}[k]$ and $b_{0}[k]$ are not Kronecker delta functions but the most recent data values $(y[k])$.

\subsection{Calculation of Fourier Transform}

The conventional DFT and its implementation, the FFT operate only in a block by block manner. The application has to wait for a block of $N$ incoming data samples to calculate the $N$-point spectrum, and refresh the results of previous measurements. This causes delays during the operation, while real-time applications need a solution which provides an $N$-wide spectrum following each incoming data sample.

To provide continuous spectrum calculation, the Sliding DFT (S-DFT) is proposed [8], which is a filter bank based on a Lagrange structure. The S-DFT uses a moving average filter which includes complex resonators. The resonator modulates the signal by $\mathrm{e}^{\mathrm{j} 2 \pi \frac{i}{N}}$, that also gives the $i$-th spectral component at $i / N$ center frequency, where the sampling frequency is unity. If $N$ filters are used, then the total $N$-point spectrum is given by the filter bank, and the result refreshes at each incoming data. The poles of this S-DFT are located on the unit circle, so the system is only marginally stable. If the applied arithmetic precision is finite, then the absolute value of resonators can be slightly smaller or larger than unity. If the poles are located outside the unit circle, the system is not going to be stable, the results may diverge. If the poles are located inside the unit circle, then the results will converge to zero [6], [8], [9].

An alternative solution for the continuous spectrum calculation is the R-DFT [6], [9], which is based on the observer theory. This model supposes that the measured signal $(x[k])$ is a linear combination of the elements of a basis system. The generated signal can be expressed as:

$$
x[k]=\sum_{i=0}^{N-1} \mathbf{X}_{i}[k] \mathbf{c}_{i}[k]
$$

where $N$ is the number of filters, $\mathbf{c}[k]$ is a basis system and $\mathbf{X}[k]$ are the weighting factors of the linear combination, which values are stored into the discrete integrators as initial values.

The observer (Fig. 4), which is responsible for the signal decomposition, has the following state variables: $\widetilde{\mathbf{X}}[k]=$ $\left(\widetilde{\mathbf{X}}_{0}[k], \widetilde{\mathbf{X}}_{1}[k], \ldots, \widetilde{\mathbf{X}}_{N-1}[k]\right)^{T}$, and its system equation can be expressed as [6]:

$$
\widetilde{\mathbf{X}}[k+1]=\widetilde{\mathbf{X}}[k]+\mathbf{g}[k] \mathbf{c}^{T}[k](\mathbf{X}[k]-\widetilde{\mathbf{X}}[k])
$$

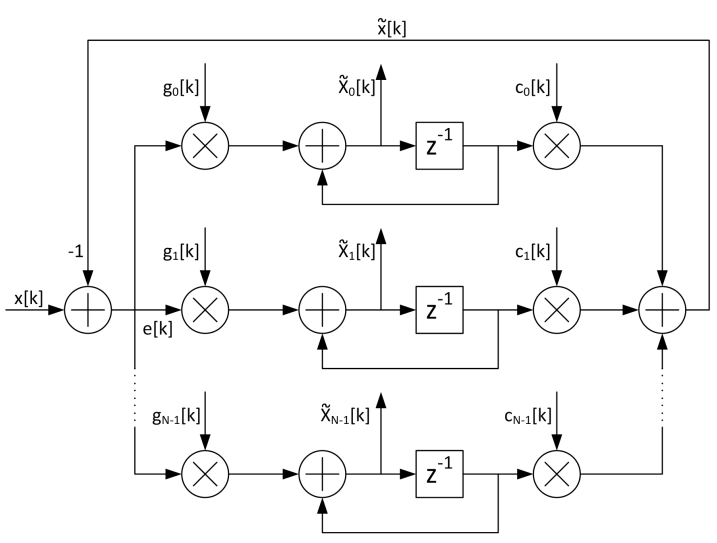

Fig. 4. Schematic structure of the observer-based R-DFT.

where $\mathbf{g}[k]$ is a basis system $\mathbf{c}[k]$. The states of the observed system can be calculated as [6]:

$\mathbf{X}[k+1]-\widetilde{\mathbf{X}}[k+1]=\prod_{\kappa=0}^{k}\left(\mathbf{I}-\mathbf{g}[\kappa] \mathbf{c}^{T}[\kappa]\right)(\mathbf{X}[0]-\widetilde{\mathbf{X}}[0])$

where I is the identity matrix. The error of the observer $(e[k])$ is given as:

$$
e[k]=\sum_{i=0}^{N-1}\left(\mathbf{X}_{i}[k]-\widetilde{\mathbf{X}}_{i}[k]\right) \mathbf{c}_{i}[k]
$$

The following 3 statements about R-DFT can be proven [6], [7]:

1. the observer is convergent, i.e. the error reaches zero in $N$ steps if $\prod_{k=0}^{N-1}\left(\mathbf{I}-\mathbf{g}[k] \mathbf{c}^{T}[k]\right)=0$, which is true if $\mathbf{c}[k]$ and $\mathbf{g}[k]$ are basis/reciprocal basis systems;

2. the state variables of the observer $(\widetilde{\mathbf{X}}[k])$ are the spectral components of the observed signal, if $\mathbf{g}_{i}[k]=$ $\frac{1}{N} \mathrm{e}^{\mathrm{j} 2 \pi \frac{k i}{N}}$ and $\mathbf{c}_{i}[k]=\mathrm{e}^{\mathrm{j} 2 \pi \frac{k i}{N}}$, so this observer calculates recursively the DFT;

3. this observer structure is equivalent to the Lagrange structure.

\subsubsection{Comparison of FFT and R-DFT}

If the DFT is applied, then the most suitable method has to be chosen for the spectrum calculation. In this section, hardware requirements are compared. These parameters have to be taken into consideration before choosing the most suitable DFT calculation method.

The input data are complex-valued, but the adders and multipliers operate with real numbers. A complex addition requires only two real adders. The multiplication is more complex: it requires five real adders and three real multipliers. To store a complex number, two real memory cells are needed. The following requirements are calculated taking into account these values, and the applied arithmetic units are considered as real-valued. 
In this paper, one of the most efficient solution to calculate FFT, the Split-Radix FFT is taken for comparison. Its requirements are proportional to $N \log _{2} N$. By contrast, the R-DFT (Fig. 4) shows a linear dependence on $N$. It contains in each branch two complex multipliers, a complex adder and a complex memory cell. In addition, there is a complex adder at the input, and $N-1$ complex adders at the output. The requirements for FFT and R-DFT are compared in Fig. 5 for $N=2^{4} \ldots 2^{16}$. It can be seen, that the requirements are about equal at $N=512$. Below 512, the FFT has the better condition, but above 512 the R-DFT becomes better and its requirements increase lower.

The FFT butterfly has $\log _{2} N$ stages, which is equal to its latency. In contrast, the R-DFT has only a single stage, and latency is induced only by the pipelined structure of the arithmetic units in a branch. The calculations of R-DFT run parallel on different branches. However, the R-DFT has a disadvantage that it contains an adder with $N$ inputs, which causes the biggest difficulty of the implementation. That is why the listed values are rather theoretical, the exact values may differ in real hardware implementation.

\subsection{Hardware Complexity of Channel Estima- tion Procedures}

The methods presented in this paper operate in the most effective way if they are implemented in FPGA or ASIC. In this case the complexity of the procedures shall be estimated not on a software basis, but based on hardware-related requirements.

In this paper, two different ways are presented for channel estimation: the correlation-based with complex valued Golay correlator and the Fourier transformation-based with R-DFT $(N=512)$. Their requirements are shown in Tab. 3 for complex valued calculations with real valued arithmetic units. These values are theoretical, the requirements may differ based on the specific implementation. FGC has much lower complexity (and it can only be used for channel impulse response estimation) than the R-DFT, however R-DFT can be used for additional synchronization and spectra estimation purposes as well, allowing a more flexible receiver operation.

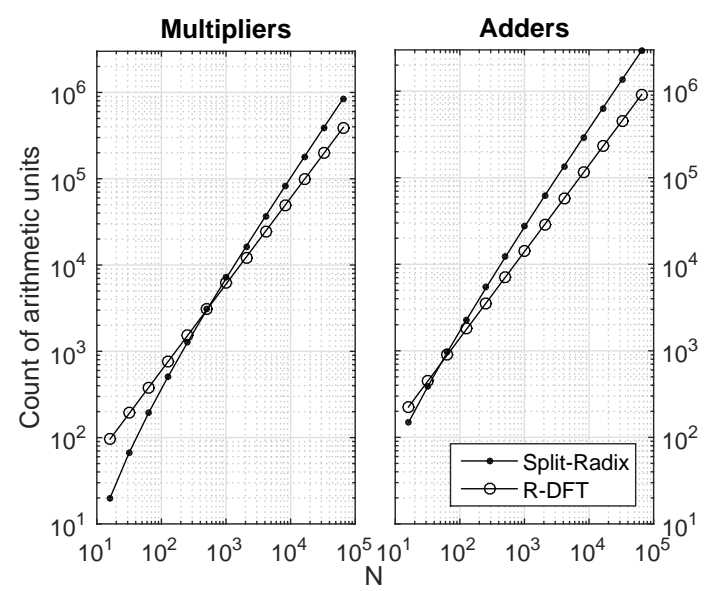

Fig. 5. Hardware requirements of DFT calculations depending on $N$.

\begin{tabular}{|c|c|c|}
\hline & Golay correlator & R-DFT \\
\hline Multiplier & 21 & 3072 \\
\hline Adder & 63 & 7168 \\
\hline Memory cell & 254 & 1024 \\
\hline
\end{tabular}

Tab. 3. Theoretical hardware requirements.

\section{Simulations}

In this section, the results of the simulations are presented. Two different channel models are applied. The first is a living room environment at $60 \mathrm{GHz}$ by Maltsev [10]. The second is the COST 207 channel model for rural area with 4 taps [12]. The structures of the two simulated baseband systems are shown in Fig. 7. The effect of the channel's noise is simulated by adding normally distributed random complex values to the transmitted data as described previously. During the simulations the sampling frequency is normalized to unity, furthermore, perfect synchronization and no frequency difference between transmitter and receiver are assumed.

\subsection{Living Room Environment at $60 \mathrm{GHz}$}

In this simulation a 802.11ad standard [11] compliant packet is used as transmit signal with preamble and header parts (where the applied modulation and coding scheme (MCS) field is 6). The size of the transmitted data is $128 \mathrm{kB}$. The payload contains cyclic prefix, and the MMSE-method is applied for channel equalization. The compensation filter (with a length of 64 taps) is generated by the Golay correlator, as well as by the R-DFT, and finally the Bit Error Rate (BER) is calculated for both cases before LDPC-decoding.

During the simulations, SNR is set from $0 \mathrm{~dB}$ in steps of $1 \mathrm{~dB}$ until BER value of $10^{-6}$ is reached. At each SNR-value, 200 packets are generated, and for each of them the BER is calculated. After the compensations, the BER is calculated for $128 \mathrm{kB}$ data. The results of the simulations are presented in Fig. 6 in function of the SNR.

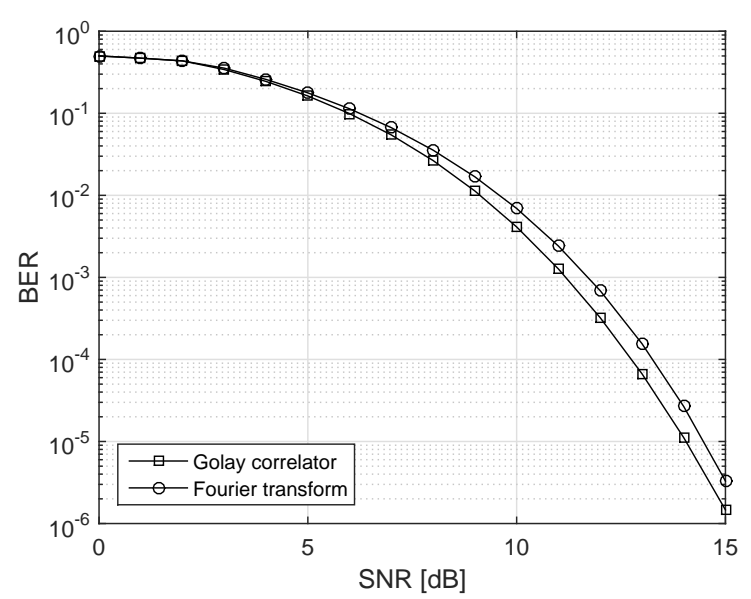

Fig. 6. BER of transmission for $N=128$-long Golay sequences (channel model: Living Room Environment at $60 \mathrm{GHz}$ ). 


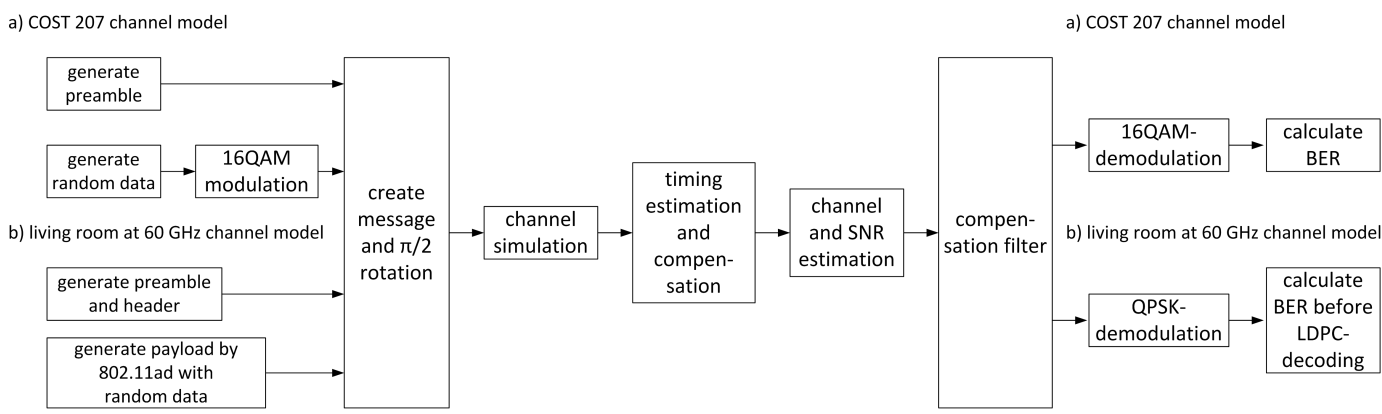

Fig. 7. Structure of the simulation.

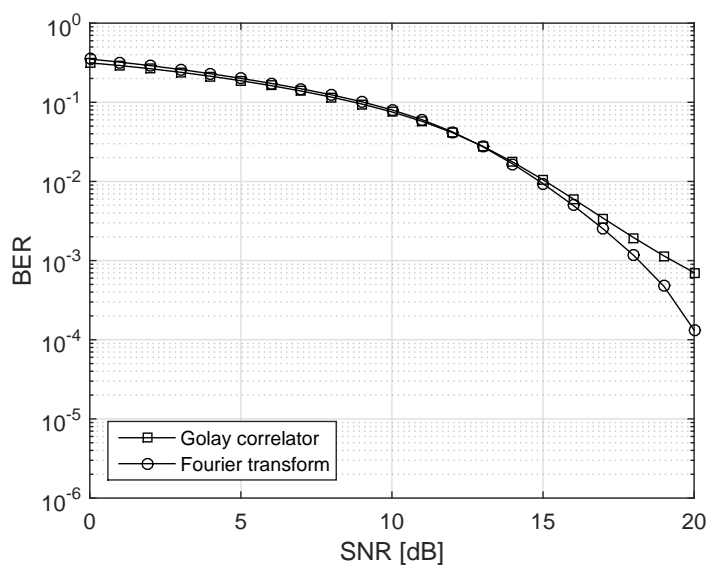

(a) 16-long Golay sequences.

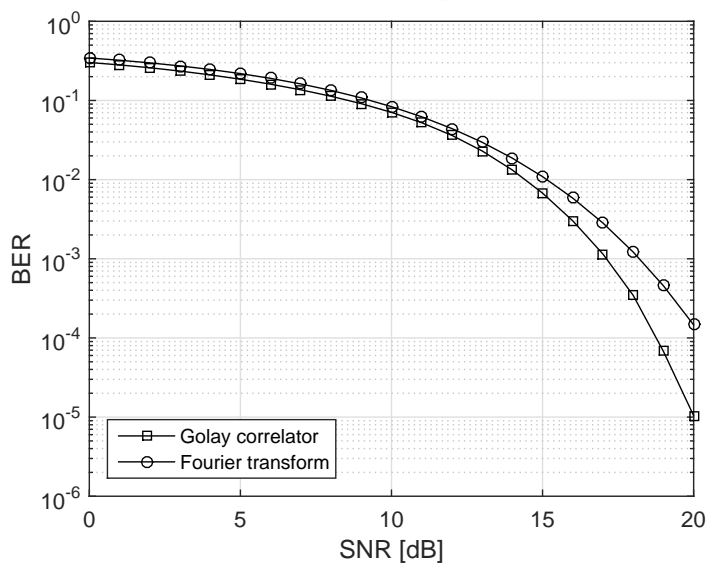

(c) 64-long Golay sequences.

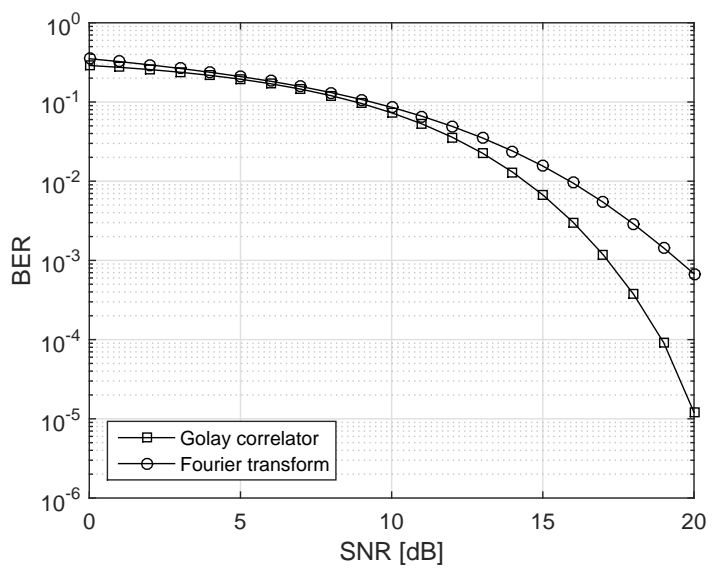

(b) 32-long Golay sequences.

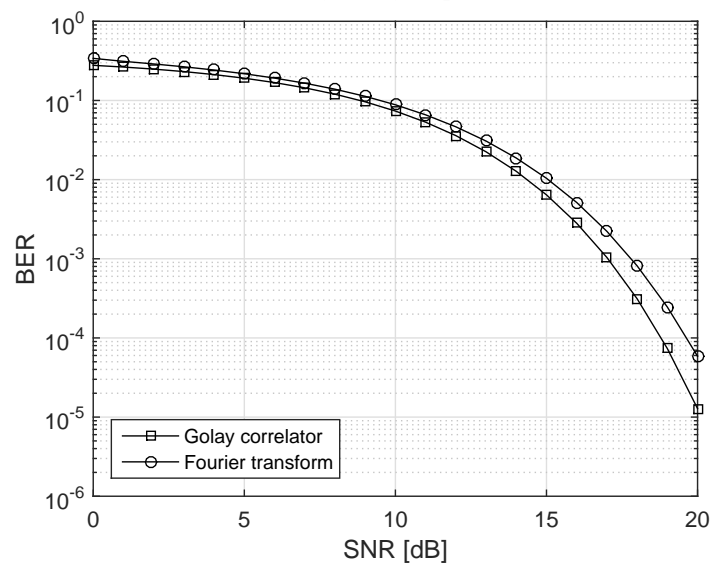

(d) 128-long Golay sequences.

Fig. 8. BER for Golay sequences with different length (channel model: COST 207).

\subsection{COST 207 Channel Model}

In this simulation scenario the transmitted packet contains a preamble with the channel estimation field (see in Sec. 2.1) and $512 \mathrm{kB}$ random data. The data field does not contain any cyclic prefix, and the ZF-method is applied for channel equalization. The compensation filter (with the half length of the applied Golay sequence) is generated by the Golay correlator, as well by the R-DFT, and finally the BER is calculated for both cases.

During the simulations, SNR is set between $0 \mathrm{~dB}$ and $20 \mathrm{~dB}$ in steps of $2 \mathrm{~dB}$ increments. At each SNR-value, 500 transmissions are generated, and for each of them the BER is calculated. After the compensations, the BERs are calcu- lated for $512 \mathrm{kB}$ data. These BER results are averaged, and the results of the simulations are presented in Fig. $8 \mathrm{a}-8 \mathrm{~d}$.

\subsection{Evaluation of the Simulations}

The results of the simulations show that both the Golay correlator and the Fourier transform are efficient solutions for CIR estimation. The complex extension of the Golay sequences makes it very suitable for such transmissions, where complex signals are applied (e.g. OFDM or a simple QAM modulation). The BER of Golay correlation depends on the ratio of lengths of the applied Golay sequences and the ideal CIR. If this ratio is low, then the BER is higher, as Fig. 8a shows, but if the ratio is higher, then the BER becomes lower 
as well (see in Fig. 8a-8d). The Golay correlator gives better CIR results thus the resulting BER is lower, but there are cases where the Fourier transform may give better result if the applied Golay sequence is short (see Fig. 8a).

This ratio influences the BER of the Fourier transform as well, but the length of the Golay sequences have another effect: the resolution of the transform $(N)$ depends on this length. If the resolution is better, then more information is given about the spectrum of transfer channel and the compensation is more accurate.

The frequency domain approach and the application of the R-DFT has further advantages compared to the time domain approach. The Golay correlator is specified for the channel estimation, as long as the DFT can be applied to estimate other parameters as well (e.g. frequency offset error). Furthermore, many transmission systems use OFDM modulation, where a DFT unit is applied to encode/decode the data, so the Golay correlator would be an additional block for the CIR estimation. Also, the message can contain cyclic guard interval [11], which is a single $g_{A}$ sequence in this case. The estimated parameters can be further improved by these guard intervals using the Fourier transform method, because the Golay correlator requires not only a $g_{A}$ but a $g_{B}$ as well to obtain the difference signal of the correlated sequences for the estimation.

\section{Conclusion}

In this paper, a time domain and a frequency domain approach are presented for CIR estimation based on complimentary Golay sequences. Hardware solutions for both methods are investigated and compared as well: a complexvalued Golay correlator is described for the time domain approach and a less known method for the calculation of the DFT - the R-DFT - is introduced which can outperform the conventional technique, the FFT. The proposed methods are compared through BER simulations using WiGig and COST 207 channel models.

\section{Acknowledgments}

This work was supported by the János Bolyai Research Fellowship of the Hungarian Academy of Sciences.

\section{References}

[1] GONG, G., HUO, F., YANG, Y. Large zero autocorrelation zones of Golay seqeunces and their applications. IEEE Transactions on Communications, 2013, vol. 61, no. 9, p. 3967-3979. DOI: 10.1109/TCOMM.2013.072813.120928

[2] DONATO, P. G., FUNES, M. A., HADAD, M. N., et al. Optimised Golay correlator. Electronics Letters, 2009, vol. 45, no. 7, p. 380-381. DOI: $10.1049 / \mathrm{el} .2009 .2923$
[3] LI, S., YUE, G., CHENG, X., et al. A novel and robust timing synchronization method for SC-FDE $60 \mathrm{GHz}$ WPAN systems. In Proceedings of the 14th IEEE International Conference on Communication Technology. Chengdu (China), 2012, p. 262-267. DOI: 10.1109/ICCT.2012.6511226

[4] WU, H., LIN, P., CHEN, K. A novel frequency offset estimation algorithm with wide range and high accuracy for OFDM system. Journal of Networks, 2014, vol. 9, no. 8, p. 2218-2223. DOI: 10.4304 /jnw.9.8.2218-2223

[5] LEI, M., HUANG, Y. CFR and SNR estimation based on complementary Golay sequences for single-carrier block transmission in 60-GHz WPAN. In Proceedings of the IEEE Wireless Communications and Networking Conference. Budapest (Hungary), 2009, p. 1-5. DOI: 10.1109/WCNC.2009.4917623

[6] CSUKA, B., KOLLÁR, I., KOLLÁR, Z., et al. Comparison of signal processing methods for calculating point-by-point discrete Fourier transforms. In Proceedings of the 26th International Conference Radioelektronika. Košice (Slovakia), 2016, p. 217-221. DOI: 10.1109/RADIOELEK.2016.7477394

[7] VARGA, L., KOLLÁR, Z. Low complexity FBMC transceiver for FPGA implementation. In Proceedings of the 23rd International Conference Radioelektronika. Pardubice (Czech Republic), 2013, p. 219-223. DOI: 10.1109/RadioElek.2013.6530920

[8] JACOBSEN, E., LYONS, R. The sliding DFT. IEEE Signal Processing Magazine, 2003, vol. 20, no. 2, p. 74-80. DOI: 10.1109/MSP.2003.1184347

[9] VARGA, L., KOLLÁR, Z., HORVÁTH, P. Recursive discrete Fourier transform based SMT receivers for cognitive radio applications. In Proceedings of the 19th International Conference on Systems, Signals and Image Processing. Vienna (Austria), 2012, p. 130-133. ISBN: 978-1-4577-2191-5

[10] MALTSEV, A., ERCEG, V., PERAHIA, E., et al. Channel Models for $60 \mathrm{GHz}$ WLAN Systems. 152 pages. [Online] Cited 2016-01-07. Available at: https://mentor.ieee.org/802.11/dcn/09/11-09-0334-0800ad-channel-models-for-60-ghz-wlan-systems.doc

[11] IEEE STANDARD FOR INFORMATION TECHNOLOGY. Local and Metropolitan Area Networks - Specific Requirements Part 11: Wireless LAN Medium Access Control (MAC) and Physical Layer (PHY) Specifications Amendment 3: Enhancements for Very High Throughput in the $60 \mathrm{GHz}$ Band. IEEE Std 802.11ad-2012. 628 pages. [Online] Cited 2016-01-07. Available at: http://ieeexplore.ieee.org/document/6392842/

[12] COST 207. Digital Land Mobile Radio Communications (Final Report). 404 pages. ISBN: 92-825-9946-9

\section{About the Authors ...}

Barna CSUKA was born in 1991. He is received his M.Sc. from the Budapest University of Technology and Economics in 2016, where he is currently a Ph.D. student. His research interests include digital signal processing, measurement theory and digital data transmission.

Zsolt KOLLÁR was born in 1983. He received his Ph.D. degree in electric engineering from the Budapest University of Technology and Economics in 2013. He joined the Rohde \& Schwarz Reference Laboratory in 2007, where he is currently a development engineer. His research interests include digital signal processing, channel coding and wireless communication. 